\title{
Breathing affects self-other voice discrimination in a bodily state with increased otherness
}

\author{
Pavo Orepic ${ }^{1}$, Hyeong-Dong Park ${ }^{1}$, Giulio Rognini ${ }^{1}$, Nathan Faivre ${ }^{2,}$, Olaf Blanke ${ }^{1,3,{ }^{*}}$ \\ 1 \\ Laboratory of Cognitive Neuroscience, Center for Neuroprosthetics and Brain Mind Institute, \\ Faculty of Life Sciences, Swiss Federal Institute of Technology (EPFL), Switzerland \\ 2 \\ Laboratoire de Psychologie et Neurocognition (LPNC), CNRS UMR 5105, Université Grenoble \\ Alpes, France \\ 3 \\ Faculty of Medicine, University of Geneva, Geneva, Switzerland \\ * equal contribution
}

Corresponding author

Olaf Blanke

Bertarelli Chair in Cognitive Neuroprosthetics, Center for Neuroprosthetics \& Brain Mind Institute, School of Life Sciences, Campus Biotech, Swiss Federal Institute of Technology (EPFL), 1012 Geneva, Switzerland

E-mail: olaf.blanke@epfl.ch

Tel: +41(0)216936921

Fax: +41 (0)216936922 


\begin{abstract}
A growing number of studies have focused on identifying cognitive processes that are modulated by interoceptive signals. Here we investigated whether interoception affects self-processing, by assessing changes in self-voice perception as a function of respiratory and cardiac cycles. Considering the fundamental role interoception plays in bodily self-consciousness, we additionally applied conflicting sensorimotor stimulation inducing a state characterized by a loss of self and increased otherness, and investigated its effects in self-other voice perception. Our data reveal that breathing, but not heartbeat, affects self-voice perception, by showing that participants $(N=30)$ discriminated self-voice from other voices better during inspiration, while being in the state of increased otherness and especially when hearing voices of other people. Loudness judgement of equivalent self-related stimuli was unaffected by breathing. Combining interoception and voice perception with self-monitoring framework, these data extend recent findings on breathing-dependent cognition to self-processing.
\end{abstract}

\title{
Keywords
}

breathing, interoception, self-voice, self-other voice discrimination, bodily self-consciousness, selfmonitoring, somatic passivity 


\section{Statement of Relevance}

Respiration and heartbeat signals, traditionally placed under the umbrella of autonomic interoceptive processing, have recently been investigated in cognitive neuroscience and identified as contributors to various cognitive processes. Compared to numerous reports relating cognition to cardiac cycle, breathingphase dependency has been demonstrated only for a few cognitive functions, and interestingly, revealed a consistent advantage of the inspiration phase. Here, we combined psychophysics with robotics and voicemorphing technology to investigate the impact of respiration and heartbeat cycles on self-voice perception and on bodily self-processing. Our data associate breathing to self-voice perception by demonstrating an inspiration-driven advantage in self-other voice discrimination, which is further affected by experimentallyinduced alterations in bodily self-perception. These findings are of broad relevance because they describe breathing-dependent effects in self-processing and thereby intersect three seemingly distinct branches of modern neuroscience - self-monitoring, voice processing and interoception. 


\section{Introduction}

Interoception refers to the processing of afferent sensory signals originating from the inside of the body, such as signals from the heart, lungs, or intestines. Although traditionally considered as mostly unconscious signals of the autonomic nervous system, recent research has shown that heartbeat and respiration signals can also affect many perceptual and cognitive processes. For instance, there is consistent evidence showing that external sensory stimuli (visual, tactile or auditory) are perceived differently when presented in different phases of the cardiac cycle (Birren, Cardon, \& Phillips, 1963; Motyka et al., 2019; Sandman, McCanne, Kaiser, \& Diamond, 1977). Cardiac phase dependency has also been observed for pain perception (Wilkinson, McIntyre, \& Edwards, 2013) and emotional processing (Garfinkel et al., 2014), as well as cognitive functions such as memory (Pfeifer et al., 2017) or social cognition (Azevedo, Garfinkel, Critchley, \& Tsakiris, 2017). By comparison, only few recent studies demonstrated respiratory phase dependency in emotion distinction and memory recall (Zelano et al., 2016), as well as visuospatial perception (Perl et al., 2019), and visual pattern recognition (Nakamura, Fukunaga, \& Oku, 2018). Interestingly, all studies investigating the impact of respiration on cognitive functions reported enhanced performance during inspiration as compared to the phase of expiration, arguably due to inspiration-driven neural synchronization of task-relevant cortical and subcortical regions (Heck, Kozma, \& Kay, 2019; Perl et al., 2019; Zelano et al., 2016).

Interoceptive signals are also known to be constituting brain mechanisms of bodily self-consciousness (BSC) (Park \& Blanke, 2019; Seth \& Tsakiris, 2018), as based on multisensory and sensorimotor bodily signals (Blanke, Slater, \& Serino, 2015). This is corroborated by experimental evidence demonstrating that the integration of conflicting interoceptive and exteroceptive signals (afferent sensory signals encompassing vision, audition, somatosensation, gustation and olfaction) can lead to altered states of BSC (Adler, 
Herbelin, Similowski, \& Blanke, 2014; Aspell et al., 2013; Suzuki, Garfinkel, Critchley, \& Seth, 2013). Similar alterations of BSC have also been reported by using a robotic device (Hara et al., 2011), which creates sensorimotor conflicts between a participant's upper limb movements and touch sensations on the back. Namely, poking movements performed with the front part of the robotic device (placed in front of participants) are replicated by the back part of the device (Figure 1), resulting in the corresponding tactile stimuli on participants' back (synchronous stimulation). Moreover, adding a temporal delay between the participants' movements and the tactile stimulation delivered on the back (asynchronous stimulation) induces an alteration of BSC characterized by differences in self-location (Blanke et al., 2014) and in selfmonitoring (Faivre et al., 2020). Importantly, these and related stimulations also induce the feeling that another person is in the room (Blanke et al., 2014; Salomon et al., 2020), a loss of self-agency (Sato \& Yasuda, 2005; Tsakiris, Haggard, Franck, Mainy, \& Sirigu, 2005), and somatic passivity (i.e. the impression that someone else is applying tactile sensations on our body), compatible with an altered state of BSC, characterized by misperceiving self as other (Blanke et al., 2014; Salomon et al., 2020).

Compared to its well-established relationship to bodily self-processing, the impact of interoceptive processes on the perception of self-related stimuli (such as perception of one's own voice or face) has only rarely been investigated (Ambrosini, Finotti, Ruben, Tsakiris, \& Ferri, 2019). Accordingly, it is not known whether inspiration-enhanced performance extends to the perception of self-related stimuli (such as perception of one's own face or voice) and whether this is further modulated by sensorimotor stimulations modulating BSC (e.g. faster responses in a self-face recognition task occurring only in an altered state of BSC). Here, we investigated cardiac and respiratory phase dependency of self-voice perception. We recorded heartbeat and respiration signals of healthy participants performing two self-related auditory tasks (self-other voice discrimination; loudness judgment) (Orepic, Rognini, Kannape, Faivre, \& Blanke, 2020). We investigated whether self-voice perception would differ in trials occurring during different parts 
of respiratory (inspiration, expiration) and heartbeat (systole, diastole) cycles. Following previously reported breathing effects on cognition (Nakamura et al., 2018; Perl et al., 2019; Zelano et al., 2016) and heartbeat effects on self-face perception (Ambrosini et al., 2019), we predicted better performance in auditory tasks during inspiration and during systole. Additionally, we explored the effects of BSC modulations on respiration and cardiac phase dependency in self-voice perception. Simultaneously with performing the auditory tasks, participants manipulated a robotic device known to induce BSC alterations through sensorimotor stimulation (Hara et al., 2011). Based on our previous findings (Blanke et al., 2014; Salomon et al., 2020), we investigated whether conflicting sensorimotor stimulation able to induce systematic changes in conscious self-other experience would modify self-other voice discrimination selectively dependent on respiratory and cardiac cycles. 


\section{Method}

\section{Participants}

The study involved 30 right-handed participants ( 9 male, mean age \pm SD: $21.8 \pm 2.4$ years old), chosen from the general population, fluent in French and naïve to the purpose of the study. All participants reported no hearing deficits and no history of psychiatric or neurological disorders. Participants gave informed consent in accordance with institutional guidelines (protocol 2015-00092, approved by the Comite Cantonal d'Ethique de la Recherche of Geneva) and the Declaration of Helsinki, and received monetary compensation (CHF 20/h). Two participants were excluded from the analysis due to noisy ECG and respiration signals. The analysis reported here was done on the sample from experiment 1 of our previous study (Orepic et al., 2020).

\section{Auditory tasks}

Pairs of gender-matched acquaintances participated in this study. Participants' voices were recorded while saying 10 words in French (Zoom H6 Handy recorder; see supplementary material for an overview). Background noise removal and normalization of the recordings for average intensity (-12 dBFS) and duration (500 milliseconds) was done in Audacity software. These recordings were used to generate voice morphs spanning a voice identity continuum between two participants (acquaintances) by using TANDEMSTRAIGHT (Kawahara, Morise, Banno, \& Skuk, 2013) (e.g. a voice morph can be generated such that it contains $30 \%$ of person A's, $70 \%$ of person B's voice). Finally, the generated voice morphs were recreated six times, such that each copy contained different sound intensity. Participants heard the recordings through a speaker (JBL Control 1 Pro) placed 1 meter behind them. The experimental design was created in MATLAB 2017b with Psychtoolbox library (Kleiner et al., 2007) 
Participants performed two auditory tasks - self-other task and loudness task. During both tasks, blindfolded participants repeatedly heard the same word twice, while the first word in each word-pair always sounded the same ( $50 \%$ self-voice, $-12 \mathrm{dBFS}$ ). In the self-other task, the second word was always equally loud as the first word (-12 dBFS), but varied in participants' self-voice percentage (\% self-voice: 15 , $30,45,55,70,85)$. In each trial, participants were instructed to indicate which of the two words sounded more like their own voice by clicking on a button. In the loudness task, the second word always contained the same ratio of the two voices ( $50 \%$ of both participants), but varied in sound intensity (dBFS: $-14,-13,-$ $12.5,-11.5,-11,-10)$. Accordingly, participants were instructed to choose the louder of the two words. Six sound intensity levels and six voice ratios were chosen based on extensive pilot testing.

\section{Robotic system}

The robotic system consisted of two integrated units: the front part - a commercial haptic interface (Phantom Omni, SensAble Technologies) - and the back part - a three degree-of-freedom robot (Hara et al., 2011) (Figure 1). Participants were seated between the front and back robot and were asked to perform repeated poking movements with their right index finger using the front robot. Participants' pokes were replicated by the back robot, thus applying corresponding touches on participants' backs. The touches were mediated by the robot either in synchronous (without delay) or asynchronous (with 500 milliseconds delay) fashion, creating different degrees of sensorimotor conflict between the upper limb movement and somatosensory feedback on the back (Blanke et al., 2014; Faivre et al., 2020; Salomon et al., 2020). Participants carried out a familiarization session, after which they were asked to perform poking movements in any direction (touches could be applied on their backs in a region with a $200 \mathrm{~mm} \times 250 \mathrm{~mm}$ surface). 


\section{Experimental design}

The study consisted of two experimental sessions. For the first session, participants came with an acquaintance (a friend), who also participated in the study. Both of them were screened for eligibility criteria, after which their voices were recorded. For the second session, each participant came individually and performed the auditory tasks. Respiration and heartbeat signals were recorded throughout the entire second session.

The second session comprised two blocks of each auditory task (loudness and self-other) - one block with the synchronous and another block with the asynchronous stimulation (Figure 1). The order of blocks (loudness synchronous, loudness asynchronous, self-other synchronous, self-other asynchronous) was counterbalanced across participants. Each block started with 60 seconds of robot manipulation, without auditory stimulation, after which an auditory cue indicated the beginning of the actual auditory task. Throughout the auditory tasks, participants continued moving the robot and auditory stimuli were not time-locked to participants' movements. Each block contained 60 randomly ordered trials (10 word pairs, each presented with 6 stimulus intensities). The words within a pair were separated by 500 milliseconds and an inter-trial interval of 1 to 1.5 seconds (randomly jittered) was added to avoid predictability of the stimuli.

At the end of the second session, participants performed two additional blocks (synchronous and asynchronous) in which they passively listened to the same voice morphs while manipulating the robot. Instead of performing an auditory task during these blocks, participants were asked to fill out a short 
questionnaire after each block (Likert scale from 0 (not at all) to 6 (very strong), adapted from Blanke et al., 2014) to assess illusory self-touch ("I felt as if I was touching my back by myself"), somatic passivity ("I felt as if someone else was touching my back") and the feeling of a presence ("I felt as if someone was standing close to me"). The questionnaire contained five additional items related to the perception of vocal stimuli, which are reported in the supplementary material.

\section{SENSORIMOTOR STIMULATION}
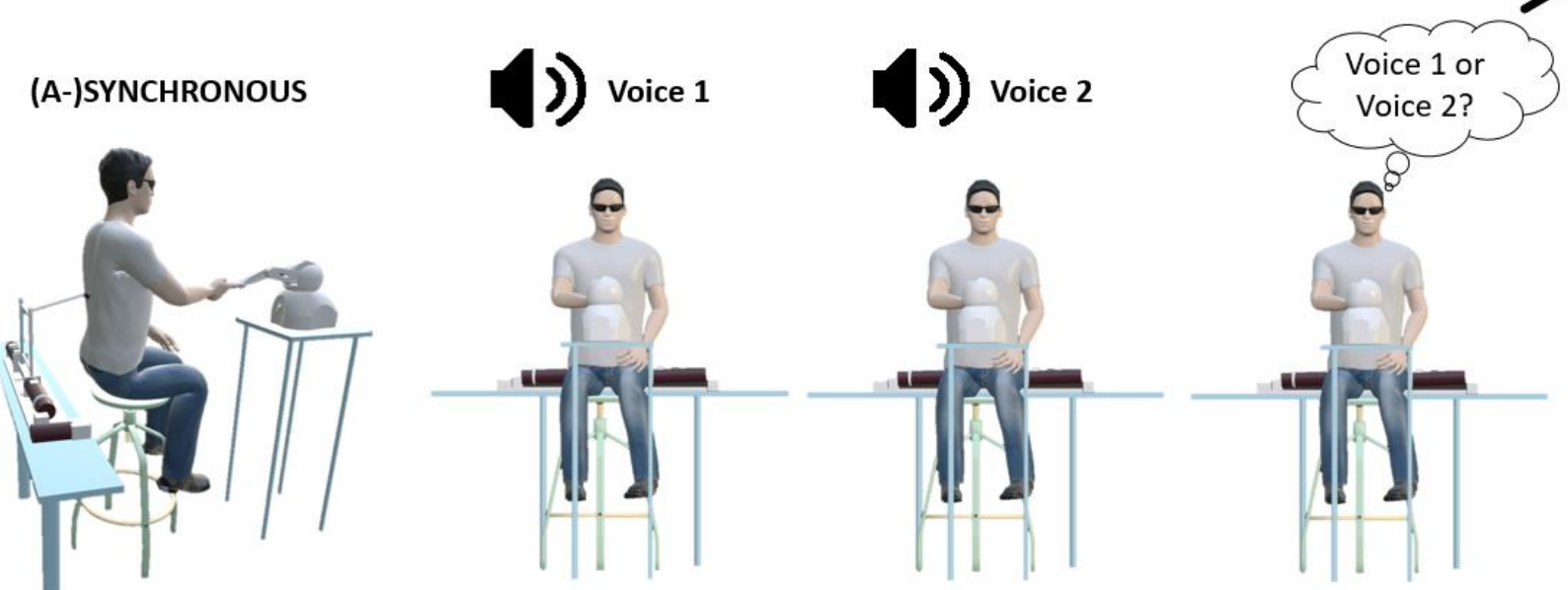

Heartbeat:

Respiration:
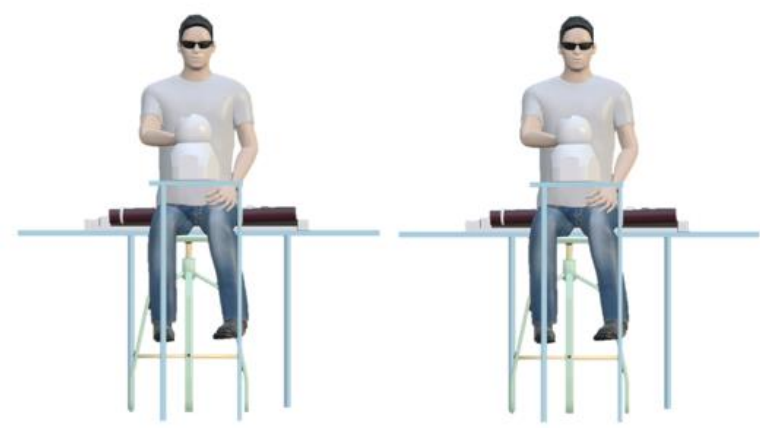

Self-other task [\% self-voice]: Loudness task [dBFS]:

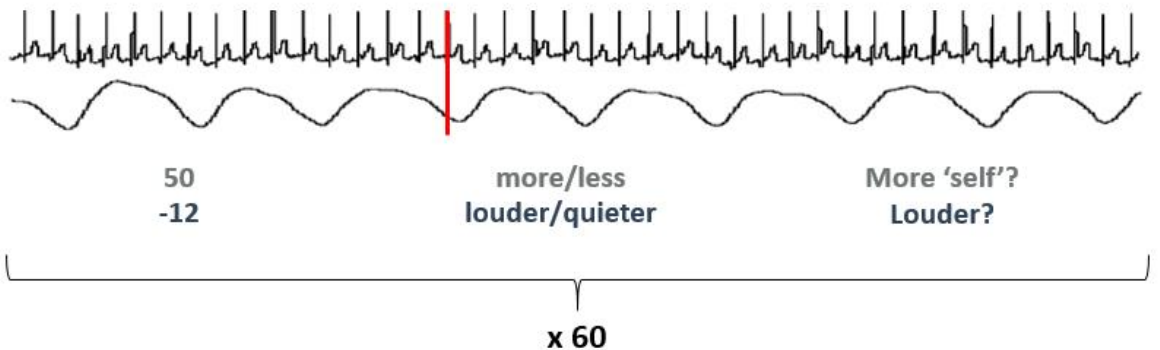

$60 \mathrm{sec}$

$$
\text { x } 60
$$

Fig. 1. Experimental block design. Heartbeat and respiration signals were continuously recorded during sensorimotor stimulation and auditory tasks (see main text for description). Cardiac and respiratory phase was extracted at the onset of the second vocal stimulus (red line). Adapted from (Orepic, Rognini, Kannape, Faivre, \& Blanke, 2020).

\section{Respiration and heartbeat}

Respiration and heartbeat signals were collected using a respiration belt and bipolar ECG electrodes (Biopac MP36R system), respectively, at a sampling rate of $2000 \mathrm{~Hz}$. Respiration belt was placed about $5 \mathrm{~cm}$ below 
participants' armpits, whereas the bipolar electrodes were placed on the 2 clavicles and lower left rib. A trigger was sent at the onset of the second word in each word pair (Figure 1), in order to determine, for each trial, in which part of the heartbeat and respiration cycle the auditory stimulus occurred.

Cycle of the continuous respiration signal was divided into inspiration and expiration periods. We first obtained signal phase values by applying Hilbert transform to the bandpass-filtered signal between 0.2 and $0.8 \mathrm{~Hz}$. Phase values belonging to the interval $(-\pi, 0)$ were classified as expiration, whereas those in the interval $(0, \pi)$ as inspiration. Systole and diastole of a heartbeat signal were defined as parts of a heartbeat cycle with previously defined onsets and durations relative to $R$ peak (Kunzendorf et al., 2019). Preprocessing of both physiological signals was conducted using the FieldTrip toolbox (Oostenveld, Fries, Maris, \& Schoffelen, 2011).

\section{Statistical analysis}

The effect of respiration on performance in both auditory tasks was analyzed with mixed-effects binomial regressions with Response as dependent variable and Respiration (inspiration, expiration), Condition (synchronous, asynchronous) and Stimulus (levels: 1-6), together with a three-way interaction, as fixed effects. The Response-variable indicates whether participants perceived a stimulus as sounding more like their own voice (self-other task) or as louder (loudness task) compared to the reference stimulus. Random effects included a by-subject random intercept. By-subject random slopes for the main effects were added following model selection based on maximum likelihood. Trials with reaction times greater or smaller than two interquartile ranges from the median for each subject were considered as outliers and excluded. 
The same mixed-effects binomial regression was applied to investigate the effect of heartbeat on auditory task performance, except that instead of the Respiration variable, the model contained Heartbeat variable with two levels: systole and diastole. A linear mixed-effects regression with Reaction Times as a dependent variable and the same fixed and random effects was also performed for both auditory tasks and both physiological signals.

The effect of Condition (synchronous, asynchronous) on ratings in questionnaire items was assessed by one-tailed t-tests, as the direction of the effect is known from previous work (Blanke et al., 2014; Salomon et al., 2020). For the questionnaire items that significantly differed between the two sensorimotor conditions (synchronous, asynchronous) on the group level, we further created a variable (e.g. Passivity variable), indicating whether individual participants experienced the illusion assessed by the corresponding question. Thus, participants were divided in two groups - those with a positive asynchronous-synchronous rating difference (e.g. Passivity+) and those with a negative or zero difference (e.g. Passivity-). Such variables were added as an additional fixed factor in the mixed-effects logistic regression assessing auditory task performance, to investigate potential effects of the subjective experience evoked by sensorimotor stimulation. Therefore, these regressions contained dependent variable Response and fixed effects of Respiration (inspiration, expiration), Stimulus (levels 1-6) and Illusion (+, -).

Finally, to control for the effects of sensorimotor stimulation and the auditory tasks on the measured physiological signals, we computed each participant's respiration and heartbeat rate and rate variability and for all four parameters performed a two-way ANOVA with Condition (synchronous, asynchronous) and Task (self-other, loudness) as fixed effects with an interaction term and by-subject random effects. Heart rate variability was represented as root mean square of successive RR interval differences (RMSSD) (Shaffer 
\& Ginsberg, 2017), and respiration rate variability as a coefficient of variation (CV) (Noto, Zhou, Schuele, Templer, \& Zelano, 2018).

To investigate potential confounds due to gender (there were more female participants), we repeated the main mixed-effects regressions with an additional main effect of Gender. However, there was no effect of Gender nor an interaction with other main effects (supplementary material) and no differences to the main results. Respiration parameters were computed using BreathMetrics (Noto et al., 2018) and heartbeat using BioSig (Schölgl, Vidaurre, \& Sander, 2011) toolbox. Statistical tests were performed with R, using notably the Ime4 (Bates, Mächler, Bolker, \& Walker, 2015), ImerTest (Kuznetsova, Brockhoff, \& Christensen, 2018), and afex (Singmann, Bolker, Westfall, \& Aust, 2019). The results were illustrated in R using sjplot (Lüdecke, 2018) and ggplot2 (Wickham, 2016) packages. 


\section{Results}

\section{Respiratory phase}

\section{Self-other discrimination}

A mixed-effects binomial regression assessing the dependence of Response to the effects of Respiration, Condition and Stimulus in the self-other discrimination task revealed a main effect of Respiration (estimate $=-1.02, \mathrm{Z}=-3.49, \mathrm{p}<0.001$ ), indicating a lower rate of 'self' response during inspiration, compared to expiration. We further observed a main effect of Stimulus (estimate $=0.53, Z=4.84, p<0.001$ ), showing that 'self' responses were more frequent when voice-morphs contained more self-features. The effect of Respiration significantly interacted with the effect of Stimulus (estimate $=0.24, Z=3.17, p=0.002$ ), revealing a steeper slope for the curve fitted for the inspiration phase. This indicates that participants were better in discriminating their own voice from another person's voice during inspiration compared to expiration

(Figure 2). By fitting four-parameter psychometric curves, we additionally showed that points of subjective equivalence (PSE) and right asymptotes did not differ between the two curves, indicating that respiration did not introduce a bias in self-other voice discrimination and did not significantly improve recognition of self-dominant stimuli (supplementary material).

The mixed-effects binomial regression also revealed a two-way interaction between the effects of Respiration and Condition (estimate $=0.89, \mathrm{Z}=2.17, \mathrm{p}=0.03$ ) and a three-way interaction between the effects of Respiration, Condition and Stimulus (estimate $=-0.21, Z=-1.97, p=0.048$ ). To further investigate the nature of these interactions, we performed separate mixed-effects logistic regression for the two levels of Condition (synchronous and asynchronous). For the dataset containing the asynchronous experimental 


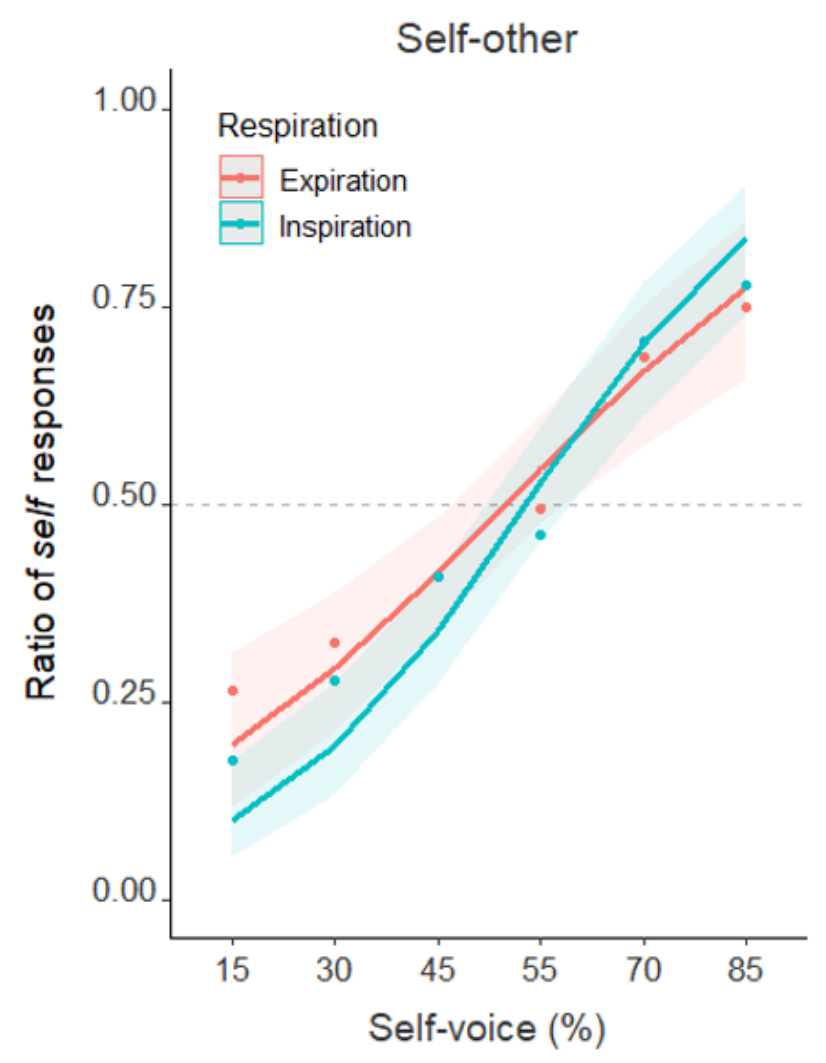

Fig. 2. Psychometric curves fitted for two respiration phases (expiration, inspiration) during the self-other task. Six stimulus levels on the abscissa represent six self-voice ratios and the ordinate indicates the rate at which the corresponding stimulus level was perceived as more resembling the 'self' than the baseline ( $50 \%$ self-voice). The dots represent grand average response. The shaded areas around each curve represent the $95 \%$ confidence intervals. A steeper curve fitting the perception during inspiration indicates that participants were better at discriminating between their own and someone else's voice during inspiration compared to expiration periods. This was especially prominent for other-dominant voice morphs.

blocks, the effect of Respiration was significant (estimate=-0.98, $Z=-3.30, p<0.001$ ) and it significantly interacted with the effect of Stimulus (estimate $=0.22, Z=2.92, p=0.004$ ), again indicating a lower intercept and a steeper slope for the inspiration phase (Figure 3, left). On the contrary, such an effect of Respiration did not occur during synchronous sensorimotor stimulation (estimate=-0.08, $Z=-0.27, p=0.79$ ) nor did it interact with the effect of Stimulus (estimate $=0.02, Z=0.22, p=0.83$ ) (Figure 3, right). This analysis shows that the observed effects of the respiration on the self-other discrimination (Figure 2) were only found during asynchronous sensorimotor stimulation (Figure 3, left) - i.e. participants were better in discriminating their own from another person's voice during the asynchronous sensorimotor stimulation, but not during the synchronous stimulation. Equivalent models relying on the Bayesian framework revealed 
evidence in favor of the null hypothesis according to which breathing did not affect self-other discrimination in the synchronous condition (Bayes factor $=0.17$, see supplementary material).
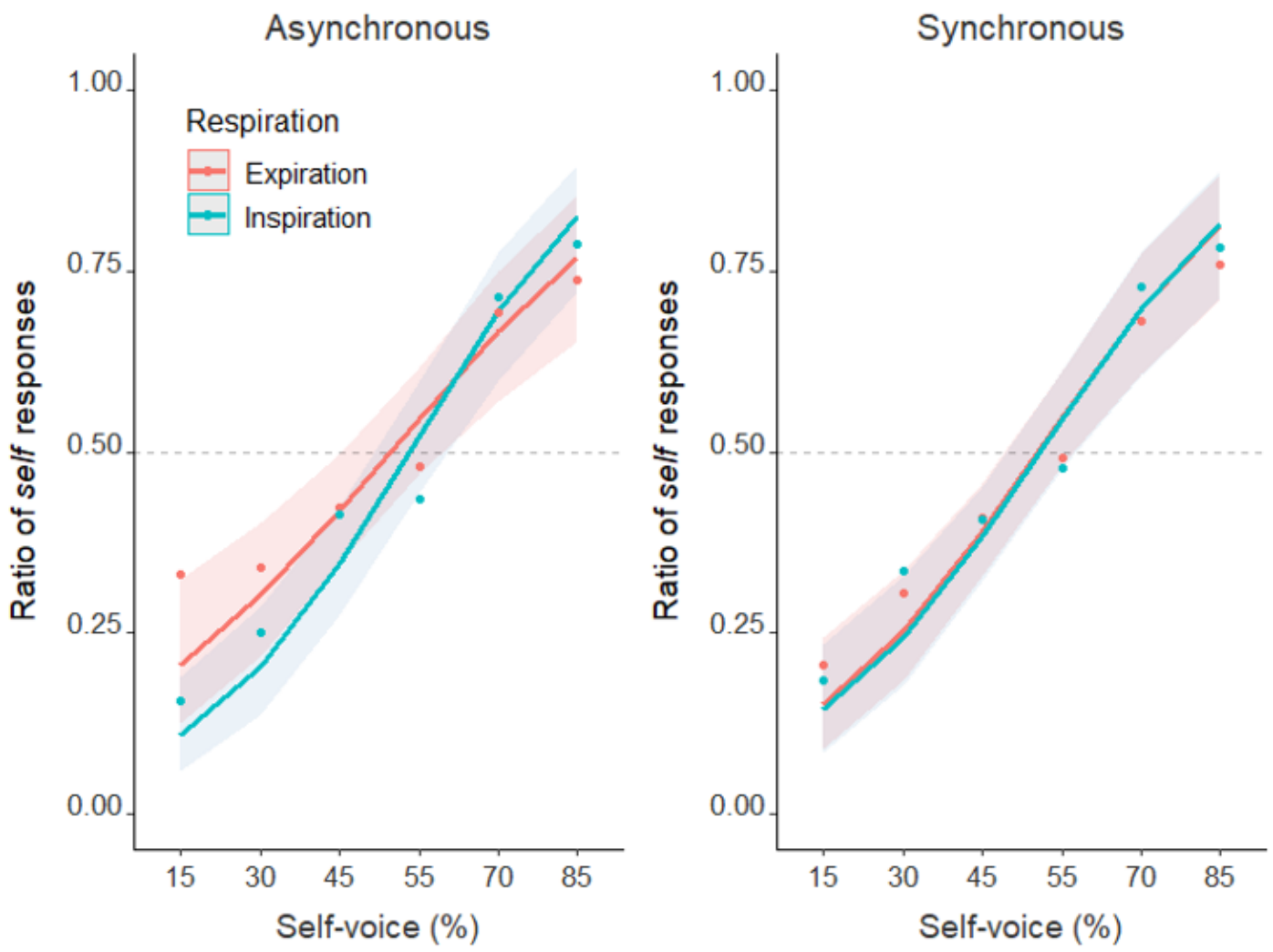

Fig. 3. Psychometric curves fitted for respiration phases for the two sensorimotor conditions of the self-other task. Inspiration was advantageous for self-other voice discrimination only during the asynchronous stimulation.

\section{Bodily self-consciousness}

As we reported previously (Orepic et al., 2020), the analysis of subjective ratings revealed that participants experienced stronger somatic passivity in the asynchronous (mean rating $=3.21,95 \% \mathrm{Cl}=[2.34,4.09]$ ) versus synchronous $(2.43,[1.57,3.28])$ condition $(t(27)=2.05, p=0.025$, Cohen's $d=0.35)$. Participants rated illusory self-touch significantly stronger in the synchronous $(2.39,[1.58,3.21])$ versus asynchronous (1.39, $[0.61,2.18])$ condition $(t(27)=2.58, p=0.008, d=0.49)$. There were no significant differences between conditions in other questionnaire items (all $p>0.05$ ). 
To further assess the relationship between the observed differences in subjective experience and in auditory perception, we ran the same mixed-effects logistic regression on self-other task performance with an additional binary independent variable reflecting whether participants experienced somatic passivity and self-touch (see methods). Thus, participants were divided in two groups - those with a positive asynchronous-synchronous rating difference (Passivity+, $N=16$ / Self-touch+, $N=14$ ) and those with a negative or zero difference (Passivity-, $N=12$ / Self-touch-, $N=14$ ). We observed a significant interaction between Passivity and Respiration (estimate $=-1.08, Z=-2.76, p=0.006$ ) and a three-way interaction between Respiration, Stimulus and Passivity borderlined with significance (estimate=0.17, Z=1.73, p=0.084; other details of the model in the supplementary material). Investigation of these interactions revealed a steeper curve for the inspiration phase only in the Passivity+ group (Figure 4, left) (Respiration: estimate=-1.18, Z=-
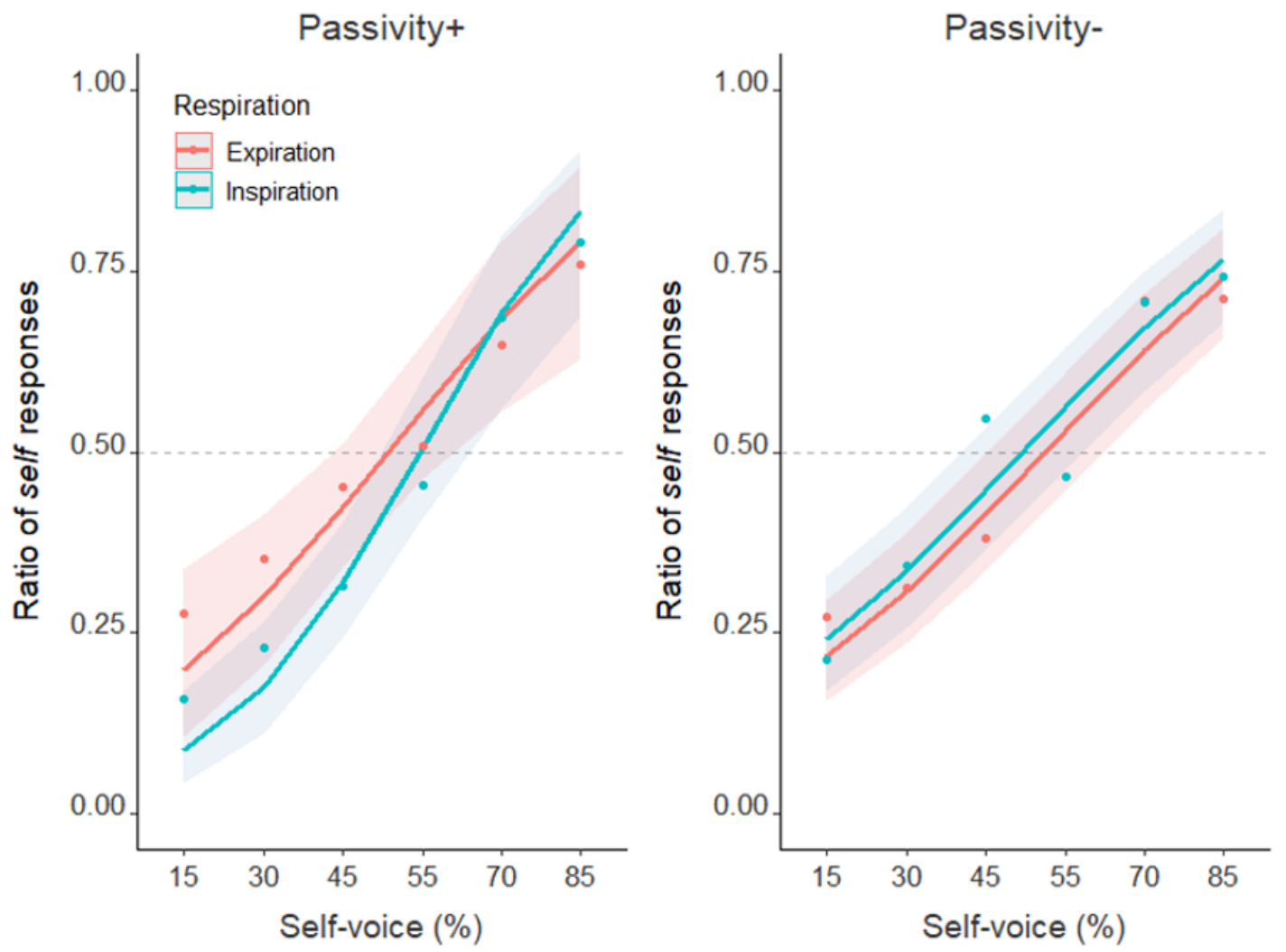

Fig. 4. Only participants experiencing somatic passivity (Passivity+, left) were better in discriminating between self and other voices during inspiration compared to expiration. Respiration did not affect voice perception in the Passivity- group (right). 
4.15, $p<0.001$; Stimulus: estimate $=0.54, Z=3.94, p<0.001$; Respiration-Stimulus interaction: estimate $=0.24$, $Z=3.32, p<0.001$ ). No such effects were found in the Passivity- group (Figure 4, right) (Respiration: estimate $=0.13, \quad Z=0.46, \quad p=0.646$; Stimulus: estimate=0.47, $Z=9.65, \quad p<0.001 ;$ Respiration-Stimulus interaction: estimate $=0, Z=0.02, p=0.984)$. There were no significant interactions between Self-touch and Respiration (supplementary material).

\section{Loudness perception}

Mixed-effects binomial regression on loudness perception with Response as a dependent variable and Respiration, Condition and Stimulus as fixed effects revealed only a main effect of Stimulus (estimate=0.56, $Z=11.73, p<0.001$ ). Respiration did not affect loudness judgement (estimate $=0.07, Z=0.28, p=0.780$ ), nor did it interact with any of the other fixed effects (Condition: estimate $=-0.25, Z=-0.68, p=0.497$; Stimulus: estimate $=0, Z=0.03, p=0.977$ ). There was no main effect of Condition (estimate $=-0.19, Z=-0.74, p=0.460$ ), no two-way interaction between Condition and Stimulus (estimate=0.07, $Z=1.01, p=0.310$ ), nor a threeway interaction between Respiration, Condition and Stimulus (estimate $=0.02, Z=0.22, p=0.825$ ). These results suggest that loudness judgement does not depend on the respiration phase (Figure 5). Equivalent Bayesian models revealed evidence in favor of the null hypothesis according to which breathing did not affect loudness judgments ( $\mathrm{BF}=0.15$, see supplementary material). 


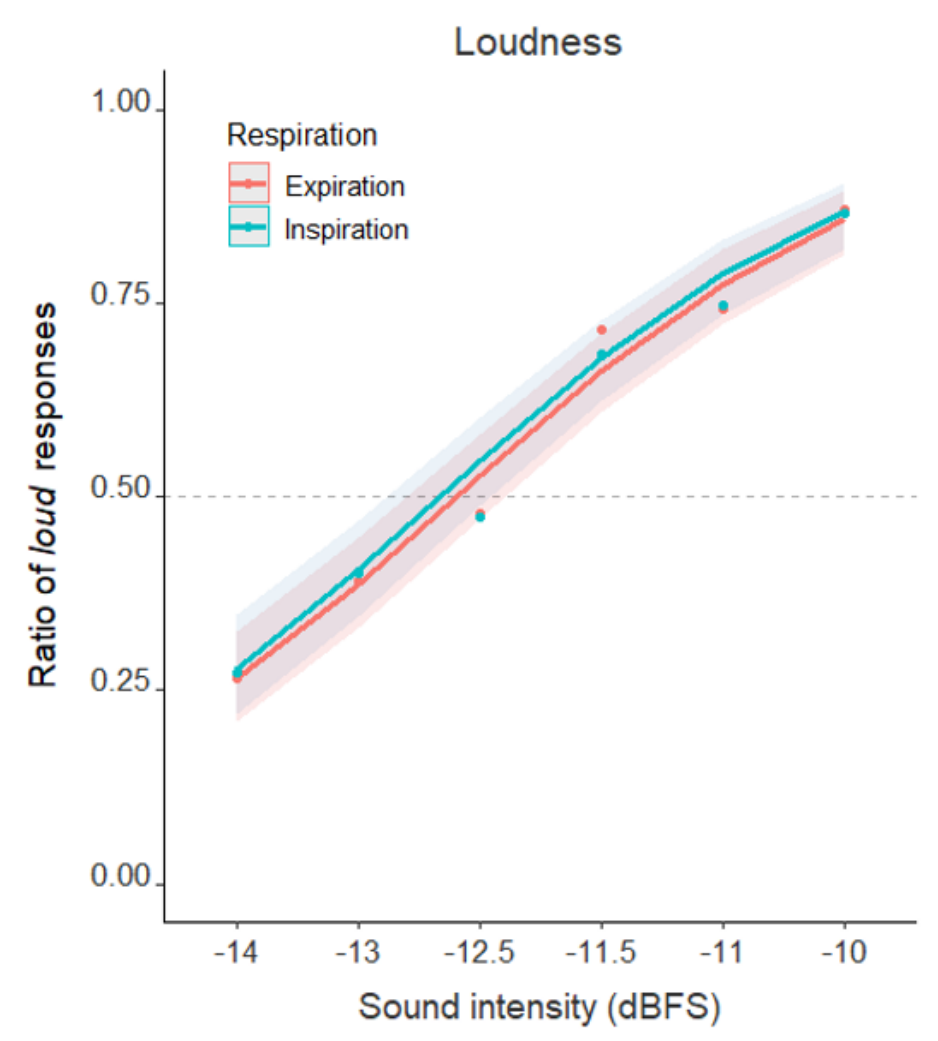

Fig. 5. Psychometric curves fitted for two respiration phases (expiration, inspiration) during the loudness task. Six stimulus levels on the abscissa represent six sound intensity levels and the ordinate indicates the rate at which the corresponding stimulus level was perceived louder than the baseline (12 dBFS). The dots represent grand averages and the shaded areas around each curve represent the $95 \%$ confidence intervals. Respiration did not affect loudness judgement.

\section{Cardiac phase}

Heartbeat phase did not affect task performance in either of the auditory tasks - there were no main effects of Heartbeat either on self-other (estimate $=0.26, z=0.79, p=0.428$ ), nor on loudness judgement (estimate $=-$ 0.27, $\mathrm{z}=-0.92, \mathrm{p}=0.359)$. Both models indicated a main effect of Stimulus (self-other: estimate $=0.56$, $Z=10.08, p<0.001$; loudness: estimate $=0.53, Z=10.12, p<0.001$ ) and no effect of Condition (self-other: estimate $=0.21, Z=0.69, p=0.488$; loudness: estimate $=-0.49, Z=-1.73, p=0.083$ ). There were no significant interactions between the fixed effects in either task (for a detailed report see supplementary material). Equivalent Bayesian models revealed evidence in favor of the null hypothesis according to which heartbeat 
did not affect self-other discrimination ( $B F=0.17$ ) nor loudness judgments ( $B F=0.26$, supplementary material).

\section{Reaction times}

There were no significant effects of Respiration on reaction times in either auditory task (loudness: estimate $=0, \mathrm{t}(105.1)=-0.2, \mathrm{p}=0.839$; self-other: estimate $=0.01, \mathrm{t}(3194)=0.79, \mathrm{p}=0.432)$. Similarly, we observed no main effect of Hearbeat in either auditory task (loudness: estimate=0, t(2491)=-0.03, p=0.976; self-other: estimate $=0.02, \mathrm{t}(2423)=0.92, \mathrm{p}=0.358)$. Finally, neither of the two effects (Respiration, Heartbeat) showed a significant interaction with other fixed effects (supplementary material).

\section{Physiological analysis}

Neither robotic stimulation nor the auditory tasks modulated parameters of respiratory or cardiac physiological signals. ANOVA did not indicate significant differences between respiration rate during asynchronous $($ mean $=0.35 \mathrm{~Hz}, 95 \% \mathrm{Cl}[0.33,0.37] \mathrm{Hz})$ and synchronous $(0.35,[0.33,0.37] \mathrm{Hz})$ conditions $(F(1,26)=0.06, p=0.813)$ and also did not differ during loudness $(0.35,[0.33,0.37] \mathrm{Hz})$ and self-other $(0.35$, $[0.33,0.37] \mathrm{Hz})$ tasks $(F(1,26)=0.12, p=0.733)$. Similarly, respiration rate variability did not differ between these conditions (asynchronous $(0.37,[0.31,0.43] \mathrm{Hz})$ vs. synchronous $(0.36,[0.30,0.42] \mathrm{Hz})$ conditions $(F(1,26)=0.21, p=0.649)$; loudness $(0.36,[0.30,0.42] \mathrm{Hz})$ vs. self-other $(0.37,[0.31,0.44] \mathrm{Hz})$ tasks $(F(1$, 26) $=0, p=0.964)$ ). This was also found for heart rate (asynchronous $(80.95,[77.36,84.55]$ bpm) vs. synchronous (81.16, [77.81, 84.51] bpm) conditions $(F(1,26)=0, p=0.945)$; loudness $(80.59,[77.20,83.99]$ bpm) vs. self-other $(81.53,[77.98,85.07] \mathrm{bpm})$ tasks $(F(1,26)=1.81, p=0.190))$ and heart rate variability (asynchronous $(36.55,[30.53,42.57] \mathrm{bpm})$ vs. synchronous $(35.09,[28.44,41.73] \mathrm{bpm})(F(1,26)=0.12$, 
$p=0.731)$; loudness $(36.18,[29.25,43.12] \mathrm{bpm})$ vs. self-other: $35.43,[29.75,41.11] \mathrm{bpm})(F(1,26)=0.13$, $p=0.717)$ ). Finally, there were no significant interactions between the effects of Condition and task for any of the parameters (breathing rate: $F(1,26)=0.62, p=0.437$; breathing rate variability: $F(1,26)=0.02$, $p=0.894$; heart rate: $F(1,26)=0, p=0.999$; heart rate variability: $F(1,26)=0.22, p=0.645)$. 


\section{Discussion}

We report that participants were better in discriminating their own from someone else's voice during inspiration compared to expiration. Moreover, this inspiratory advantage for self-voice processing was stronger during the asynchronous sensorimotor stimulation and was thus more pronounced in the condition inducing illusory misattribution of self-generated sensations to someone else. Breathing did not affect auditory perception when participants made loudness judgements of the same self-related vocal stimuli and cardiac phase did not modulate the performance in either auditory task.

An advantage of inspiration over expiration in self-other discrimination has not been reported before and extends previous respiratory phase dependency data to self-related cognition. Previously, other cognitive processes have been shown to be improved during inspiration: memory retrieval (Zelano et al., 2016), spatial perception (Perl et al., 2019), visual pattern recognition (Nakamura et al., 2018), and emotion discrimination (Zelano et al., 2016). It has been argued that the inspiratory phase of the respiratory cycle drives neural synchronization of cortical and sub-cortical regions, thereby affecting the corresponding taskrelated neural activations (Heck et al., 2019; Zelano et al., 2016). Based on differences in resting-state functional connectivity between inspiration and expiration phases, it has also been proposed that inspiration-driven patterns of neural activity may improve the processing of incoming stimuli (Perl et al., 2019). Our results demonstrate a solid inspiratory-phase advantage based on psychophysics and thereby associate breathing to self-related processing. We observed that inspiration led to systematic sensitivity changes, but no perceptual bias, in self-other voice discrimination (i.e. steeper psychometric curve, Figure 2) and that it was driven by differences in other-dominant voice morphs (left asymptote; Figure 2). It is further worth noticing that the breathing effect was observed only for self-other voice discrimination while basic breathing parameters such as breathing rate and variability were equal in both auditory tasks. These 
data demonstrate that inspiration leads to improved and unbiased self-other voice discrimination and that it does not improve loudness judgement of the same vocal stimuli, showing that inspiration only impacted aspects of auditory perception related to discriminating one's own voice from another person's voice.

Another important finding was that the improvement in self-other discrimination during inspiration was driven by changes related to online sensorimotor stimulation, and, in particular, to asynchronous sensorimotor stimulation, with basic breathing parameters (rate and rate variability) being unaffected by the stimulation itself. Thus, the observed increase in sensitivity for self-other discrimination during the inspiration phase (as indicated by a steeper psychometric curve) was specific to performance during asynchronous sensorimotor stimulation. These differences between inspiration and expiration were absent in the synchronous condition, suggesting that the stronger sensorimotor conflict impacts the effect of the breathing cycle on self-other discrimination. Namely, asynchronous stimulation contains a stronger sensorimotor conflict, as there is an additional temporal conflict between poking movements in the front and tactile sensations on participants' back, in addition to the spatial conflict, which is also present during synchronous stimulation. Such robotically-applied sensorimotor conflicts during asynchronous stimulation have been related to changes in BSC, especially to the feeling of a presence and to somatic passivity (Blanke et al., 2014; Salomon et al., 2020). The present data link the inspiration-driven advantage for self-other voice discrimination not only to asynchronous sensorimotor simulation, but also to the altered BSC state associated with it. Thus, additional analysis revealed that the inspiration-driven effect was observed only in the participants reporting somatic passivity - i.e. in those participants susceptible to misattributing selfgenerated tactile sensations to someone else. This links the present breathing effect in self-other discrimination not only to the online respiratory cycle and sensorimotor state of the participant, but also to conscious self-representation (i.e. somatic passivity). Interestingly, the advantage of inspiration in selfother voice discrimination was again most pronounced for other-dominant voice morphs, indicating that 
in an other-oriented BSC state (i.e. otherness associated during asynchronous stimulation with somatic passivity) participants were more sensitive to recognizing self-other morphs as another person. We argue that respiration and conflicting sensorimotor stimulation combine to induce systematic changes in conscious self-other experience and self-other discrimination that depend on the respiration cycle. Our findings show an improvement in self-other voice discrimination, especially while hearing the voices of another person, in individuals experiencing a loss of self and enhanced otherness, suggesting that breathing facilitates self-other discrimination only during a BSC state characterized by otherness, a shift from self towards other.

To summarize, we demonstrate (1) a relationship between breathing and self-other voice discrimination, which is (2) dependent on sensorimotor integration and (3) related to feelings of otherness in the form of somatic passivity. From the two tested interoceptive functions, only respiration, but not cardiac, phase affected self-voice perception. Breathing is fundamentally related to speech and voice production (thus to the sound of our own voice) (Von Euler, 2011) and with voluntary action (H. Park et al., 2020). We argue that the present findings about the coupling between breathing and self-other voice discrimination may reflect that voice perception and the voluntary action of speaking are coupled with the basic physiological function of breathing, which are absent (or less pronounced) for cardiac physiology. We also did not observe cardiac-dependent differences in reaction times as it has been reported for self-face perception (Ambrosini et al., 2019), arguing that different physiological signals (e.g. respiration and heartbeat) affect self-related processes differently, depending on their intrinsic cyclic differences, their specific functional associations, and likely the investigated sensory modality. Our data shed new light on the interactions between interoception, BSC and self-voice perception and as such extend previous findings on breathingdependent cognition to self-related processing. 


\section{Author contribution}

Study concept and design: PO, GR, NF, OB. Acquisition of data: PO. Analysis and interpretation of data: PO, HP, GR, NF, OB. Drafting of the manuscript: PO, HP, NF, OB. Critical revision of the manuscript for important intellectual content: All authors. Statistical analysis: PO, NF. Obtained funding: OB. Administrative, technical, or material support: All authors. Study supervision: NF, OB.

\section{Open Practices Statement}

Neither of the tasks reported in this article was formally preregistered. Anonymized data and analysis scripts are available on the open science framework (https://osf.io/kvarn/). 


\section{References}

Adler, D., Herbelin, B., Similowski, T., \& Blanke, O. (2014). Breathing and sense of self: Visuorespiratory conflicts alter body self-consciousness. Respiratory Physiology \& Neurobiology, 203, 68-74. https://doi.org/10.1016/j.resp.2014.08.003

Ambrosini, A. E., Finotti, G., Ruben, T., Tsakiris, M., \& Ferri, F. (2019). Seeing myself through my heart: cortical processing of a single heartbeat speeds up self-face recognition. Biological Psychology, 44(200), 1-30. https://doi.org/10.1093/annonc/mdy039/4835470

Aspell, J. E., Heydrich, L., Marillier, G., Lavanchy, T., Herbelin, B., \& Blanke, O. (2013). Turning Body and Self Inside Out: Visualized Heartbeats Alter Bodily Self-Consciousness and Tactile Perception. Psychological Science, 24(12), 2445-2453.

https://doi.org/10.1177/0956797613498395

Azevedo, R. T., Garfinkel, S. N., Critchley, H. D., \& Tsakiris, M. (2017). Cardiac afferent activity modulates the expression of racial stereotypes. Nature Communications, 8, 1-9. https://doi.org/10.1038/ncomms13854

Bates, D., Mächler, M., Bolker, B. M., \& Walker, S. C. (2015). Fitting linear mixed-effects models using Ime4. Journal of Statistical Software. https://doi.org/10.18637/jss.v067.i01

Birren, J. E., Cardon, P. V., \& Phillips, S. L. (1963). Reaction time as a function of the cardiac cycle in young adults. Science, 140(3563), 195-196.

https://doi.org/10.1126/science.140.3563.195-a

Blanke, O., Pozeg, P., Hara, M., Heydrich, L., Serino, A., Yamamoto, A., ... Rognini, G. (2014). Neurological and robot-controlled induction of an apparition. Current Biology, 24(22), 2681-2686. https://doi.org/10.1016/j.cub.2014.09.049

Blanke, O., Slater, M., \& Serino, A. (2015). Behavioral, Neural, and Computational Principles of Bodily Self-Consciousness. Neuron, 88(1), 145-166.

https://doi.org/10.1016/j.neuron.2015.09.029

Faivre, N., Vuillaume, L., Bernasconi, F., Salomon, R., Blanke, O., \& Cleeremans, A. (2020). Sensorimotor conflicts alter metacognitive and action monitoring. Cortex, 124(22), 26812686. https://doi.org/10.1016/j.cortex.2019.12.001

Garfinkel, S. N., Minati, L., Gray, M. A., Seth, A. K., Dolan, R. J., \& Critchley, H. D. (2014). Fear from the Heart: Sensitivity to Fear Stimuli Depends on Individual Heartbeats. Journal of Neuroscience, 34(19), 6573-6582. https://doi.org/10.1523/JNEUROSCI.3507-13.2014

Hara, M., Rognini, G., Evans, N., Blanke, O., Yamamoto, A., Bleuler, H., \& Higuchi, T. (2011). A novel approach to the manipulation of body-parts ownership using a bilateral master-slave system. In IEEE International Conference on Intelligent Robots and Systems (pp. 4664-4669). https://doi.org/10.1109/IROS.2011.6048519

Heck, D. H., Kozma, R., \& Kay, L. M. (2019). The rhythm of memory: how breathing shapes memory function. Journal of Neurophysiology, 122(2), 563-571. 
https://doi.org/10.1152/jn.00200.2019

Kawahara, H., Morise, M., Banno, H., \& Skuk, V. G. (2013). Temporally variable multi-aspect Nway morphing based on interference-free speech representations. In 2013 Asia-Pacific Signal and Information Processing Association Annual Summit and Conference, APSIPA 2013 (pp. 1-10). IEEE. https://doi.org/10.1109/APSIPA.2013.6694355

Kleiner, M., Brainard, D. H., Pelli, D. G., Ingling, A., Murray, R., \& Broussard, A Ingling, R Murray, C. (2007). What's new in Psychtoolbox-3? Perception, 36(14), 1-16. https://doi.org/10.1068/v070821

Kunzendorf, S., Klotzsche, F., Akbal, M., Villringer, A., Ohl, S., \& Gaebler, M. (2019). Active information sampling varies across the cardiac cycle. Psychophysiology, 1-16. https://doi.org/10.1111/psyp.13322

Kuznetsova, A., Brockhoff, P. B., \& Christensen, R. H. B. (2018). ImerTest Package: Tests in Linear Mixed Effects Models. Journal of Statistical Software, 82(13), 1-26. https://doi.org/10.18637/jss.v082.i13

Lüdecke, D. (2018). sjPlot: Data Visualization for Statistics in Social Science. R package version 2.6.2. https://doi.org/10.5281/zenodo.1308157

Motyka, P., Grund, M., Forschack, N., Al, E., Villringer, A., \& Gaebler, M. (2019). Interactions between cardiac activity and conscious somatosensory perception. Psychophysiology, 56(10), e13424. https://doi.org/10.1111/psyp.13424

Nakamura, N. H., Fukunaga, M., \& Oku, Y. (2018). Respiratory modulation of cognitive performance during the retrieval process. PLOS ONE, 13(9), e0204021.

Noto, T., Zhou, G., Schuele, S., Templer, J., \& Zelano, C. (2018). Automated analysis of breathing waveforms using BreathMetrics: A respiratory signal processing toolbox. Chemical Senses, 43(8), 583-597. https://doi.org/10.1093/chemse/bjy045

Oostenveld, R., Fries, P., Maris, E., \& Schoffelen, J. M. (2011). FieldTrip: Open source software for advanced analysis of MEG, EEG, and invasive electrophysiological data. Computational Intelligence and Neuroscience. https://doi.org/10.1155/2011/156869

Orepic, P., Rognini, G., Kannape, O. A., Faivre, N., \& Blanke, O. (2020). Sensorimotor conflicts induce somatic passivity and louden quiet voices in healthy listeners. BioRxiv. https://doi.org/https://doi.org/10.1101/2020.03.26.005843

Park, H., Barnoud, C., Trang, H., Kannape, O. A., Schaller, K., \& Blanke, O. (2020). Breathing is coupled with voluntary action and the cortical readiness potential. Nature Communications, 11(1), 1-8. https://doi.org/10.1038/s41467-019-13967-9

Park, H. D., \& Blanke, O. (2019). Coupling Inner and Outer Body for Self-Consciousness. Trends in Cognitive Sciences, 23(5), 377-388. https://doi.org/10.1016/j.tics.2019.02.002

Perl, O., Ravia, A., Rubinson, M., Eisen, A., Soroka, T., Mor, N., ... Sobel, N. (2019). Human nonolfactory cognition phase-locked with inhalation. Nature Human Behaviour, 3(5), 501-512. 
https://doi.org/10.1038/s41562-019-0556-z

Pfeifer, G., Garfinkel, S. N., Gould van Praag, C. D., Sahota, K., Betka, S., \& Critchley, H. D. (2017). Feedback from the heart: Emotional learning and memory is controlled by cardiac cycle, interoceptive accuracy and personality. Biological Psychology, 126, 19-29. https://doi.org/10.1016/j.biopsycho.2017.04.001

Salomon, R., Progin, P., Griffa, A., Rognini, G., Do, K. Q., Conus, P., ... Blanke, O. (2020). Sensorimotor Induction of Auditory Misattribution in Early Psychosis. Schizophrenia Bulletin. https://doi.org/10.1093/schbul/sbz136

Sandman, C. A., McCanne, T. R., Kaiser, D. N., \& Diamond, B. (1977). Heart rate and cardiac phase influences on visual perception. Journal of Comparative and Physiological Psychology, 91(1), 189. https://doi.org/10.1037/h0077302

Sato, A., \& Yasuda, A. (2005). Illusion of sense of self-agency: Discrepancy between the predicted and actual sensory consequences of actions modulates the sense of self-agency, but not the sense of self-ownership. Cognition, 94(3), 241-255. https://doi.org/10.1016/j.cognition.2004.04.003

Schölgl, A., Vidaurre, C., \& Sander, T. H. (2011). BioSig: The free and open source software library for biomedical signal processing. Computational Intelligence and Neuroscience. https://doi.org/10.1155/2011/935364

Seth, A. K., \& Tsakiris, M. (2018). Being a Beast Machine: The Somatic Basis of Selfhood. Trends in Cognitive Sciences, 22(11), 969-981. https://doi.org/10.1016/j.tics.2018.08.008

Shaffer, F., \& Ginsberg, J. P. (2017). An Overview of Heart Rate Variability Metrics and Norms. Frontiers in Public Health, 5, 258. https://doi.org/10.3389/fpubh.2017.00258

Singmann, H., Bolker, B., Westfall, J., \& Aust, F. (2019). afex: Analysis of Factorial Experiments. R package version 0.23-0. https://cran.r-project.org/package=afex. Retrieved from https://cran.r-project.org/package=afex

Suzuki, K., Garfinkel, S. N., Critchley, H. D., \& Seth, A. K. (2013). Multisensory integration across exteroceptive and interoceptive domains modulates self-experience in the rubber-hand illusion. Neuropsychologia, 51(13), 2909-2917. https://doi.org/10.1016/j.neuropsychologia.2013.08.014

Tsakiris, M., Haggard, P., Franck, N., Mainy, N., \& Sirigu, A. (2005). A specific role for efferent information in self-recognition. Cognition, 96(3), 215-231. https://doi.org/10.1016/j.cognition.2004.08.002

Von Euler, C. (2011). Brain Stem Mechanisms for Generation and Control of Breathing Pattern. In Comprehensive Physiology (pp. 1-67). https://doi.org/10.1002/cphy.cp030201

Wickham, H. (2016). ggplot2: Elegant Graphics for Data Analysis. Springer-Verlag New York. Springer-Verlag New York. Retrieved from http://ggplot2.org

Wilkinson, M., McIntyre, D., \& Edwards, L. (2013). Electrocutaneous pain thresholds are higher 
during systole than diastole. Biological Psychology, 94(1), 71-73.

https://doi.org/10.1016/j.biopsycho.2013.05.002

Zelano, C., Jiang, H., Zhou, G., Arora, N., Schuele, S., Rosenow, J., \& Gottfried, J. A. (2016). Nasal Respiration Entrains Human Limbic Oscillations and Modulates Cognitive Function. The Journal of Neuroscience, 36(49), 12448-12467. https://doi.org/10.1523/JNEUROSCI.258616.2016 


\title{
Supplementary material: Breathing affects self-other voice discrimination in a bodily state with increased otherness
}

\author{
Pavo Orepic ${ }^{1}$, Hyeong-Dong Park ${ }^{1}$, Giulio Rognini ${ }^{1}$, Nathan Faivre ${ }^{2,}$, Olaf Blanke ${ }^{1,3,{ }^{*}}$ \\ 1 \\ Laboratory of Cognitive Neuroscience, Center for Neuroprosthetics and Brain Mind Institute, \\ Faculty of Life Sciences, Swiss Federal Institute of Technology (EPFL), Switzerland \\ 2 \\ Laboratoire de Psychologie et Neurocognition (LPNC), CNRS UMR 5105, Université Grenoble \\ Alpes, France \\ 3 \\ Faculty of Medicine, University of Geneva, Geneva, Switzerland \\ * equal contribution
}

\section{Corresponding author}

Olaf Blanke

Bertarelli Chair in Cognitive Neuroprosthetics, Center for Neuroprosthetics \& Brain Mind Institute, School of Life Sciences, Campus Biotech, Swiss Federal Institute of Technology (EPFL), 1012 Geneva, Switzerland

E-mail: olaf.blanke@epfl.ch

Tel: +41 (0)216936921

Fax: +41 (0)216936922 


\section{Additional analyses}

To further specify the nature of perceptual alterations caused by the respiration phase, for each participant and each respiration phase (inspiration, expiration) we estimated four parameters of the corresponding psychometric curve - point of subjective equivalence (PSE), slope, left and right asymptote - using quickpsy (Linares \& López-Moliner, 2016) library. A two-way ANOVA with Parameter Value as a dependent variable and fixed effects Respiration (inspiration, expiration) and Parameter (PSE, slope, left and right asymptote) showed a significant interaction between the two effects $(F(1.45,39.06)=4.03, p=0.04)$. A two-tailed paired t-test was thus performed for each Parameter to assess whether its Parameter Value differed between inspiration and expiration phases. Consistent with the mixed-effects binomial regression, the curve fitted for the inspiration phase had a steeper slope $(t(27)=2.23, p=0.034$, Cohen's $d=0.61)$ and a lower left asymptote $(t(27)=-2.49, p=0.019, d=0.46)$. Additionally, there were no differences between the values of PSE $(t(27)=0.61, p=0.546, d=0.14)$ and right asymptote $(t(27)=0, p=0.995, d=0)$. These effects show that inspiration phase increased sensitivity to self-other voice discrimination (slope effect) and that the difference in performance was especially prominent for the other-dominant voice morphs (left asymptote) (main Figure 2). Respiration did not introduce a bias in self-other voice perception (absence of the PSE effect) and did not significantly improve recognition of self-dominant stimuli (right asymptote).

\section{Bayesian analyses of the null effects}

To further validate nulls findings of the mixed-effects binomial regressions reported in the main text, we ran three main regressions in a Bayesian framework: (1) for the absence of the respiration effect in loudness task, (2) for the absence of the respiration effect in the synchronous condition of the self-other task, and (3) for the absence of the cardiac effect. All Bayesian models were created in Stan computational 
framework (http://mc-stan.org/) accessed with the brms package (Bürkner, 2017), based on four chains of 10000 iterations including 2000 warmup samples. We report the highest density probability for all estimates, which specifies the range covering the $95 \%$ most credible values of the posterior estimates.

For the null effect of Respiration in the loudness task we had a prior assumption of better performance during the inspiration phase - represented by a steeper slope - (i.e., prior on the interaction between the effects of Respiration and Stimulus with Gaussian distribution of mean $=0.24$ and SD $=0.4$, based on the same interaction observed in the self-other task). No interaction between Respiration and Stimulus was found (estimate $=0.02$, highest posterior density interval $=[-0.120 .15]$, Bayes factor $=0.15$ ). Similar effects were observed even with a Gaussian prior centered on zero (estimate $=0.01, \mathrm{Cl}=\left[\begin{array}{l}-0.130 .14\end{array}\right], \mathrm{BF}=0.17$ ). Using the same prior (mean $=0.24, S D=0.4$ ), we observed no significant interaction between Respiration and Stimulus for the synchronous condition of the self-other task (estimate $=0.03, \mathrm{Cl}=[-0.110 .17], \mathrm{BF}=$ 0.17). Bayes factors smaller than 0.3 support the null hypothesis, according to which breathing did not affect loudness judgement nor self-other discrimination during the synchronous condition.

Similarly, for the cardiac phase, we had a prior assumption of a better performance during systole represented by a steeper slope - (i.e., prior on the interaction between the effects of Heartbeat and Stimulus with Gaussian distribution of mean $=0.24$ and $S D=0.4$, based on the same interaction observed for the Respiration effect in the self-other task). No interaction between Heartbeat and Stimulus was found for either task (Self-other: estimate $=0, \mathrm{Cl}=[-0.160 .15], \mathrm{BF}=0.17$; Loudness: estimate $=0.08, \mathrm{Cl}=[-0.08$ $0.23], \mathrm{BF}=0.26)$. Again, Bayes factors smaller than 0.3 support the null hypothesis of no effects of heartbeat phase on auditory tasks' performance. 


\section{Words}

As reported in our previous work (Orepic, Rognini, Kannape, Faivre, \& Blanke, 2020), participants were recorded saying 10 words in French (clou, fouet, hache, lame, lutte, os, rat, sang, scie, ver). The words were chosen from the list of 100 negatively-valenced words, as rated by 20 schizophrenic patients and 97 healthy participants (Jalenques, Enjolras, \& Izaute, 2013). Negative words were purposefully chosen in our previous study (Orepic et al., 2020), in order to better approximate the phenomenology of auditory-verbal hallucinations $(\mathrm{AVH})$, that are mostly negative in content (Woods, Jones, Alderson-Day, Callard, \& Fernyhough, 2015).

\section{Bodily self-consciousness}

An overview of the questionnaire, also reported in our previous work (Orepic et al., 2020) is given in Table S1.

Table S1. Likert-scale questionnaire used to assess subjective experience evoked by sensorimotor stimulation.

Self-touch I felt as if I was touching my back by myself.

Somatic passivity I felt as if someone else was touching my back.

Control I felt as if I had 3 bodies.

Presence hallucination I felt as if someone was standing close to me.

Friend-voice I felt as if I heard my friend's voice more often than my own voice

Friend-presence I felt as if my friend was standing close to me.

Other-presence I felt as if someone else than my friend was standing close to me

Other-voice I felt as if I heard a voice that was neither my friend's nor mine.

Bodily sensations While hearing the words, I felt changes in my body sensations (e.g. lighter, warmer, I felt tingling sensations etc.) 
As indicated and reported in the main text, for the self-other task, we ran two mixed-effects binomial regression with a dependent variable Response and fixed effects of Respiration, Stimulus and Passivity/Selftouch. Besides a significant interaction between Respiration and Passivity (see main text), the model which had Passivity as an additional factor only showed a significant effect of Stimulus (estimate=0.47, Z=9.67, $p<0.001$ ). The main effects of Passivity (estimate $=0.12, Z=0.38, p=0.704$ ) and of Respiration (estimate $=0.13$, $Z=0.46, p=0.645)$ were not significant. Stimulus did not interact significantly neither with the effect of Respiration (estimate $=0, Z=0.02, p=0.985$ ), nor with the effect of Passivity (estimate $=0, Z=-0.11, p=0.911$ ). The model with Self-touch as an additional factor also showed a significant effect of Stimulus (estimate=0.5, $Z=10.84, p<0.001$ ), whereas effects of Respiration (estimate $=-0.19, Z=-0.69, p=0.492$ ) and Self-touch were not significant (estimate $=0.47, \mathrm{Z}=1.45, \mathrm{p}=0.148$ ). This model identified no significant interactions: Stimulus and Self-touch (estimate=-0.07, $Z=-1.11, p=0.266$ ), Respiration and Self-touch (estimate=-0.5, $Z=-1.3$, $p=0.194$ ), Stimulus and Respiration (estimate $=0.07, Z=0.94, p=0.347$ ), Respiration, Stimulus and Self-touch (estimate $=0.05, \mathrm{Z}=0.51, \mathrm{p}=0.608$ ).

\section{Control analysis of the Gender effect}

To investigate potential confounds due to gender (there were more female participants), we conducted a logistic mixed-effects regression with an additional main effect of Gender. As described in the main text, the model contained Response as a dependent variable and besides the effects of Respiration (inspiration, expiration), Condition (asynchronous, synchronous) and Stimulus (levels: 1-6) - all related with an interaction term - the newly added effect of Gender (male, female) was related with the effect of Respiration with an interaction term. As model having a four-way interaction between all the fixed effects did not converge, and because the effect of Respiration was of the main interest in the study, we chose to model its interactions with all other effects, including Gender. The model showed neither a main effect of 
Gender (estimate $=0, Z=-0.02, p=0.983$ ) nor a significant interaction between Gender and Respiration (estimate $=0.13, \mathrm{Z}=0.78, \mathrm{p}=0.433$ ).

\section{Cardiac phase}

In Table S2 we report the non-significant interactions between the fixed effects in both tasks, omitted from the main text, and in Figure S1, we illustrate the lack of cardiac phase effects on the performance of both auditory tasks.

Table S2. An overview of the interactions containing fixed effect of Heartbeat in binomial mixed-effects regressions assessing performance in Self-other and Loudness tasks.

\begin{tabular}{llllc}
\hline Task & Heartbeat & estimate & Z value & p value \\
\hline Self-other & Condition & -0.4 & -0.88 & 0.377 \\
\cline { 2 - 4 } & Stimulus & -0.03 & -0.32 & 0.752 \\
\cline { 2 - 4 } & Condition * Stimulus & 0.04 & 0.36 & 0.717 \\
\hline Loudness & Condition & 0.39 & 0.92 & 0.359 \\
& Stimulus & 0.06 & 0.79 & 0.429 \\
\cline { 2 - 4 } & Condition * Stimulus & -0.06 & -0.48 & 0.63 \\
\hline
\end{tabular}



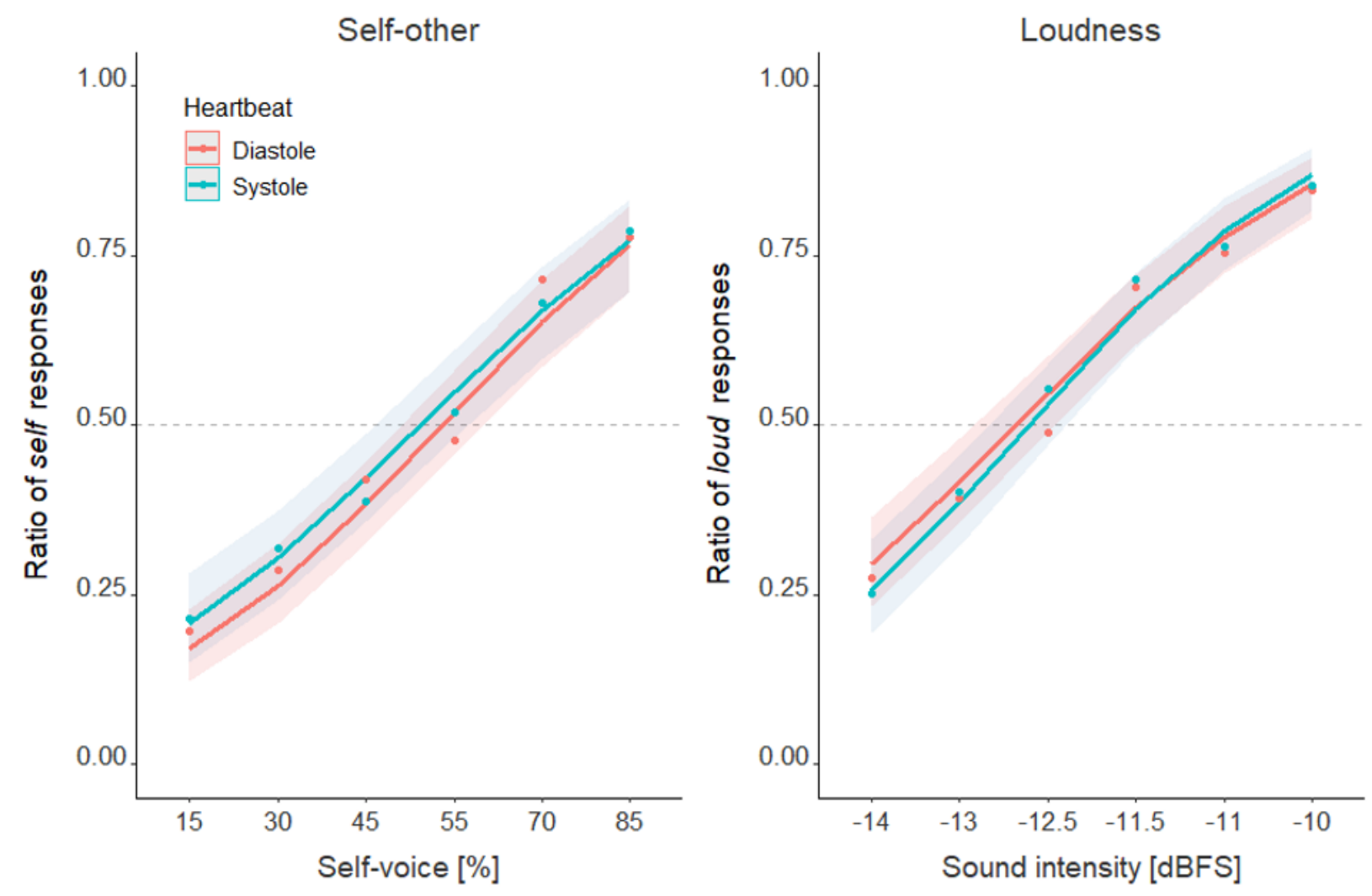

Fig. S1. Psychometric curves fitted for two heartbeat phases (systole, diastole) during self-other (left) and loudness (right) tasks. Six stimulus levels on the abscissa represent six self-voice ratios (left) and six sound intensity levels (right), whereas the ordinate indicates the rate at which the corresponding stimulus level was perceived as more resembling the 'self' (left) or as louder (right) than the baseline (50\% self-voice, $12 \mathrm{dBFS}$ ). The shaded areas around each curve represent the $95 \%$ confidence intervals. Heartbeat did not affect performance in either auditory task.

\section{Reaction times}

As main effects of Respiration and Heartbeat were reported in the main text for both auditory tasks (self-

other, loudness), here we report their interactions with other fixed effects, none of which was significant (Table S3). 
Table S3. An overview of the interactions containing fixed effects of Respiration and Heartbeat in linear mixedeffects regressions assessing response times in Self-other and Loudness tasks.

\begin{tabular}{|c|c|c|c|c|c|c|}
\hline Task & Interaction & & estimate & $\mathrm{df}$ & t value & $p$ value \\
\hline \multirow[t]{6}{*}{ Self-other } & Respiration * & Condition & 0.01 & 3203 & 0.56 & 0.577 \\
\hline & & Stimulus & -1.24 & 3191 & -0.95 & 0.342 \\
\hline & & Condition * Stimulus & -0.39 & 3192 & -0.21 & 0.832 \\
\hline & Heartbeat* & Condition & 0 & 2428 & -0.03 & 0.978 \\
\hline & & Stimulus & -1.02 & 2416 & -0.78 & 0.438 \\
\hline & & Condition * Stimulus & 1.15 & 2418 & 0.64 & 0.524 \\
\hline \multirow[t]{6}{*}{ Loudness } & Respiration * & Condition & 0 & 3185 & -0.26 & 0.797 \\
\hline & & Stimulus & 0.77 & 3152 & 0.83 & 0.408 \\
\hline & & Condition * Stimulus & -0.44 & 3157 & -0.33 & 0.739 \\
\hline & Heartbeat * & Condition & 0 & 2486 & 0.13 & 0.897 \\
\hline & & Stimulus & -0.11 & 2485 & -0.12 & 0.907 \\
\hline & & Condition * Stimulus & 0.6 & 2484 & 0.42 & 0.673 \\
\hline
\end{tabular}




\section{Supplementary references}

Bürkner, P. C. (2017). brms: An R package for Bayesian Multilevel Models using Stan. Journal of Statistical Software, 80(1).

Jalenques, I., Enjolras, J., \& Izaute, M. (2013). Valence émotionnelle des mots. L'Encéphale, 39, 189-197.

Linares, D., \& López-Moliner, J. (2016). quickpsy: An R Package to Fit Psychometric Functions for Multiple Groups. The R Journal, 8(1), 122-131. Retrieved from https://journal.rproject.org/archive/2016-1/linares-na.pdf

Orepic, P., Rognini, G., Kannape, O. A., Faivre, N., \& Blanke, O. (2020). Sensorimotor conflicts induce somatic passivity and louden quiet voices in healthy listeners. BioRxiv. https://doi.org/https://doi.org/10.1101/2020.03.26.005843

Woods, A., Jones, N., Alderson-Day, B., Callard, F., \& Fernyhough, C. (2015). Experiences of hearing voices: Analysis of a novel phenomenological survey. The Lancet Psychiatry, 2(4), 323-331. https://doi.org/10.1016/S2215-0366(15)00006-1 